\title{
CARCINOMA PROSTÁTICO: FACTORES PREQUIRÚRGICOS ASOCIADOS CON EXTENSIÓN EXTRAPROSTÁTICA Y MÁRGENES POSITIVOS HOSPITAL DE SAN JOSÉ, BOGOTÁ DC, COLOMBIA
}

\author{
Edgardo Yaspe Costa MD*, Carolina Silva Morera MD**, Patricia López Correa MD***
}

\section{Resumen}

El cáncer de próstata es causa importante de mortalidad y es invaluable la estadificación adecuada de los pacientes para minimizar las complicaciones del tratamiento quirúrgico. El objetivo es describir las características prequirúrgicas de los pacientes con extensión extraprostática y/o márgenes positivos en casos de adenocarcinomas de próstata tratados mediante prostatectomía radical abierta en el Hospital de San José de Bogotá DC, de enero 2001 a diciembre 2007. Se estudiaron 34 pacientes a quienes se les determinó el puntaje de Gleason del espécimen de prostatectomía, el TNM patológico, la presencia o no de extensión extraprostática y los márgenes quirúrgicos positivos, los cuales se relacionaron con factores prequirúrgicos como edad, niveles séricos de PSA, TNM clínico y puntaje de Gleason en biopsia. La media de edad fue 64.2 años (DE 6.7). La frecuencia de márgenes quirúrgicos positivos y extensión extraprostática fue 44 y $47 \%$. Se observó mayor compromiso de márgenes quirúrgicos y extensión extraprostática cuando el puntaje de Gleason fue de 8 ó más.

Palabras clave: próstata, cáncer de próstata, extensión extracapsular, extraprostática, puntaje de Gleason, márgenes positivos.

Abreviaturas: PSA, antígeno prostático específico; TNM, estadificación.

\section{PROSTATE CARCINOMA: PREOPERATIVE FACTORS RELATED WITH EXTRAPROSTATIC EXTENTION AND POSITIVE MARGINS HOSPITAL DE SAN JOSÉ, BOGOTÁ DC. COLOMBIA}

\begin{abstract}
Prostate cancer is a leading cause of mortality thus adequate staging is of incalculable value for minimizing surgical treatment complications. The objective of this study performed at Hospital de San José, Bogotá DC from January 2001 to December 2007 is to describe the preoperative data of patients with a prostate adenocarcinoma with extraprostatic extension and/or positive margins treated with an open radical prostatectomy. The Gleason score, pathologic TNM
\end{abstract}

Fecha recibido: agosto 3 de $201 \mathrm{I}$ - Fecha aceptado: febrero 23 de 2012

* Profesor Asistente, Fundación Universitaria de Ciencias de la Salud. Bogotá DC, Colombia.

\footnotetext{
** Residente II de Patología, Fundación Universitaria de Ciencias de la Salud Bogotá, DC, Colombia.

*** Residente III de Patología. Fundación Universitaria de Ciencias de la Salud. Bogotá, DC, Colombia.
} 
stage, presence or non presence of extraprostatic extension and positive surgical margins of the prostatectomy specimen were determined in 34 participants and related with preoperative factors such as age, PSA level, clinical TNM stage and biopsy Gleason score. The median age was 64.2 years (SD 6). The rate of positive surgical margins and extraprostatic extension was $44 \%$ and $47 \%$ respectively. A greater surgical margin compromise and extraprostatic extension was observed when the Gleason score was 8 or higher.

Key words: prostate, prostate cancer, extracapsular extension, extraprostatic, Gleason score, positive margins.

\section{Introducción}

El adenocarcinoma de próstata es la neoplasia maligna más frecuente y la segunda causa de muerte en hombres americanos y se ha estimado que representa el 33\% de todos los cánceres nuevos diagnosticados en esta población. ${ }^{1}$ Uno de cada diez hombres puede desarrollarlo y tres de cada cien es posible que mueran por dicha causa. La incidencia clínica es baja antes de los 50 años y cerca de tres cuartas partes de todos los casos ocurren en hombres de 65 años o más. ${ }^{2}$ La enfermedad ha aumentado por varios factores, entre los que se encuentran la mayor edad de la población y el perfeccionamiento de los métodos diagnósticos. Hoy día la mayoría son diagnosticados en estadios tempranos; sin embargo, a pesar de la mejoría de estos procesos, la entidad sigue un curso clínico impredecible.

Después de realizar el diagnóstico de adenocarcinoma de próstata, el clínico debe determinar si la lesión está confinada a la glándula (en potencia curable) o si se ha extendido más allá (incurable) ${ }^{3}$, para lo cual se utilizan diversos sistemas de estadificación, modelos pronósticos, nomogramas, tablas y otros elementos que tienen como finalidad la predicción lo más exacta posible del estadio de la enfermedad y del pronóstico del paciente. La medición del antígeno prostático específico (PSA) sérico, en combinación con el tacto rectal, el puntaje Gleason de la biopsia y el TNM clínico, han sido los factores prequirúrgicos más utilizados para establecer el estadio patológico del cáncer en la literatura mundial, mediante las tablas que describió Partin en 1993. ${ }^{4}$
La prostatectomía radical es el procedimiento que ofrece una mejor opción de curación de los pacientes con adenocarcinoma localizado desde el punto de vista clínico, lo cual exige la exactitud diagnóstica de los llamados "grupos de riesgo", permitiendo una indicación terapéutica acorde con la realidad de la enfermedad"; por lo tanto, la elección del tratamiento es un equilibrio entre la eficacia, las complicaciones del mismo y la calidad de vida del paciente una vez tratado.

En nuestro país se realizó un estudio similar entre 1992 y $2000^{6}$, donde se estableció que el único factor prequirúrgico asociado con extensión extraprostática y márgenes quirúrgicos positivos era el T3 clínico, lo cual difiere de lo reportado en la literatura mundial. Cabe resaltar, sin embargo, que en este trabajo la tendencia a subgraduar el puntaje Gleason de la biopsia y la multiplicidad de observadores para esta variable, pudieron haber influenciado los resultados.

El propósito de este estudio es determinar cuáles de las características prequirúrgicas se relacionan con extensión extraprostática y/o márgenes quirúrgicos positivos, con el fin de propiciar la creación de un modelo pronóstico práctico en futuras investigaciones, que ayude a la individualización y tratamiento de los pacientes con adenocarcinoma de próstata en nuestra institución.

\section{Métodos}

Se realizó un estudio descriptivo retrospectivo de 46 pacientes consecutivos, los cuales fueron diagnosticados con adenocarcinoma de próstata mediante biopsia 
transrectal por el servicio de patología del Hospital de San José de Bogotá DC. Además, se les realizó prostatectomía radical abierta entre enero 2001 y diciembre 2007 a quienes disponían de las biopsias transrectales y las piezas de prostatectomía radical para estudio histopatológico. Se excluyeron los que recibieron radioterapia preoperatoria. Del total de pacientes susceptibles de participar, ocho tenían historia clínica incompleta sin datos de niveles de PSA y/o TNM clínico; en tres no se encontraron láminas histológicas para estudio y uno que las tenía eran no valorables.

Las muestras de biopsia y prostatectomía fueron reevaluadas por un patólogo oncólogo durante el desarrollo del estudio. Para determinar el grado histológico tumoral, tanto de la biopsia como del espécimen definitivo, se siguieron las directrices de la clasificación de Gleason modificada en $2005 .^{7}$ La edad, el nivel sérico de PSA y el TNM clínico fueron tomados de la historia de los pacientes. El estadio patológico en la pieza de prostatectomía se realizó basado en el TNM patológico del año 2009. Se consideró extensión extraprostática a la presencia de células tumorales más allá de los confines de la próstata (mezcladas con tejido adiposo, comprometiendo espacios perineurales y paquetes neurovasculares más allá de la próstata o por fuera del tejido glandular prostático normal) y/o compromiso de las vesículas seminales, definida como infiltración tumoral dentro de su parénquima y no el área adventicia. Se consideraron márgenes positivos ante la presencia de células neoplásicas en contacto con los límites de transección pintados con tinta negra.

El grado histológico de la biopsia, el TNM clínico y los niveles séricos de PSA fueron correlacionados con los hallazgos de la prostatectomía radical (grado histológico, TNM patológico, extensión extraprostática y márgenes quirúrgicos positivos). Se analizaron las variables prequirúrgicas (edad, PSA, Gleason de la biopsia y TNM clínico) en concordancia con el estudio previo de Aponte y colaboradores de $2001 .{ }^{6} \mathrm{La}$ edad y los valores de PSA fueron categorizados de acuerdo con los grupos de riesgo reportados en la literatura. Para el análisis de la información se utilizó el paquete estadístico STATA 10. El protocolo fue aprobado por el Comité de Investigaciones y Ética de la Facultad de
Medicina de la Fundación Universitaria de Ciencias de la Salud y la investigación fue clasificada sin riesgo.

\section{Resultados}

En el estudio se incluyeron un total de 34 pacientes que contaban con láminas histológicas de la biopsia transrectal y del espécimen de prostatectomía para evaluación. El promedio de edad fue 64.2 años (DE 6.7). Los TNM clínicos más comunes fueron T1c $(44,1 \%)$ y T2a (38,3\%). Ningún paciente se clasificó con estadio T3 ó mayor. En la biopsia se consideraron 18 casos con puntaje de Gleason entre 2 y $6(52,9 \%)$. En la mayoría el nivel de PSA fue menor a $10 \mathrm{ng} / \mathrm{ml}(67,7 \%)$ y ninguno recibió radioterapia prequirúrgica (Tabla 1). Respecto a la información del espécimen, el TNM patológico más frecuente fue T3a (35,3\%). Diecisiete se evaluaron como Gleason 7 (50\%). Se identificó extensión extracapsular y márgenes positivos en 47 y 44,1\% (Tabla 1). El tipo histológico más común fue adenocarcinoma acinar $(94,1 \%)$; dos casos se diagnosticaron adenocarcinoma acinar más ductal $(5,9 \%)$. En los especímenes no se observaron puntajes de Gleason mayores en los pacientes de edad avanzada.

Observando la relación entre los valores de PSA y el Gleason del espécimen, trece con PSA menores de $10 \mathrm{ng} / \mathrm{ml}$ tuvieron una puntuación superior o igual a 7. Solo uno presentó PSA superior a $20 \mathrm{ng} / \mathrm{ml}$ y fue clasificado 7 (Figura 1). Llama la atención la alta frecuencia de extensión extraprostática y PSA menor de $10 \mathrm{ng} / \mathrm{ml}$ (Tabla 2).

Entre aquellos con márgenes positivos, cinco $(33,3 \%)$ tuvieron Gleason entre 8 y 10 , en contraste con los negativos donde solo uno $(5,2 \%)$ presentó puntaje entre 8 y 10 (Tabla 3 ).

Entre los pacientes con márgenes positivos cuatro cursaron con Gleason mayor de $8(26,7 \%)$ en el espécimen definitivo y no se identificó ninguno mayor de 8 en los casos con márgenes negativos (Tabla 4). Del total de pacientes solo ocho $(23,5 \%)$ presentaron extensión extraprostática y márgenes quirúrgicos positivos, que al compararlos con las variables prequirúrgicas seis 
(75\%) tenían valores de PSA menores de $10 \mathrm{ng} / \mathrm{ml}$ (50\%) y cuatro cursaron con puntajes de Gleason de 7 como se ve en la Tabla 5.

\begin{tabular}{|c|c|c|}
\hline \multicolumn{3}{|l|}{ Caracteristicas (n: 34) } \\
\hline \multicolumn{3}{|l|}{ Variables prequirúrgicas } \\
\hline Edad, años promedio (DE) & 64.2 & $(6.7)$ \\
\hline mínima - máxima & 49 & 79 \\
\hline PSA, mediana (RIQ) & 7.45 & $(5.8-12.1)$ \\
\hline \multicolumn{3}{|l|}{ Categorías PSA, n (\%) } \\
\hline$<10 \mathrm{ng} / \mathrm{ml}$ & 23 & (67.7) \\
\hline $10-20 \mathrm{ng} / \mathrm{ml}$ & 10 & $(29.4)$ \\
\hline$>20 \mathrm{ng} / \mathrm{ml}$ & 1 & (2.9) \\
\hline \multicolumn{3}{|c|}{ Gleason puntaje biopsia, n (\%) } \\
\hline $2-6$ & 18 & $(52.9)$ \\
\hline 7 & 10 & $(29.3)$ \\
\hline $8-10$ & 6 & $(17.7)$ \\
\hline \multicolumn{3}{|l|}{ TNM clínico, n (\%) } \\
\hline $\mathrm{Tla}$ & 1 & $(2.9)$ \\
\hline TIC & 15 & $(44.1)$ \\
\hline $\mathrm{T} 2 \mathrm{a}$ & 13 & $(38.3)$ \\
\hline $\mathrm{T} 2 \mathrm{~b}$ & 5 & $(14.7)$ \\
\hline \multicolumn{3}{|l|}{ Variables posquirúrgicas } \\
\hline \multicolumn{3}{|c|}{ Gleason puntaje del espécimen, $n$ (\%) } \\
\hline $2-6$ & 13 & $(38.2)$ \\
\hline 7 & 17 & $(50.0)$ \\
\hline $8-10$ & 4 & $(11.8)$ \\
\hline Márgenes positivos, n (\%) & 15 & $(44.1)$ \\
\hline Extensión extraprostática & 16 & $(47.0)$ \\
\hline \multicolumn{3}{|l|}{ TNM patológico, n (\%) } \\
\hline $\mathrm{T} 2 \mathrm{a}$ & 5 & (14.7) \\
\hline $\mathrm{T} 2 \mathrm{~b}$ & 2 & (5.9) \\
\hline $\mathrm{T} 2 \mathrm{c}$ & 11 & (32.3) \\
\hline T3a & 12 & (35.3) \\
\hline T3b & 2 & (5.9) \\
\hline T3bNI & 2 & (5.9) \\
\hline
\end{tabular}

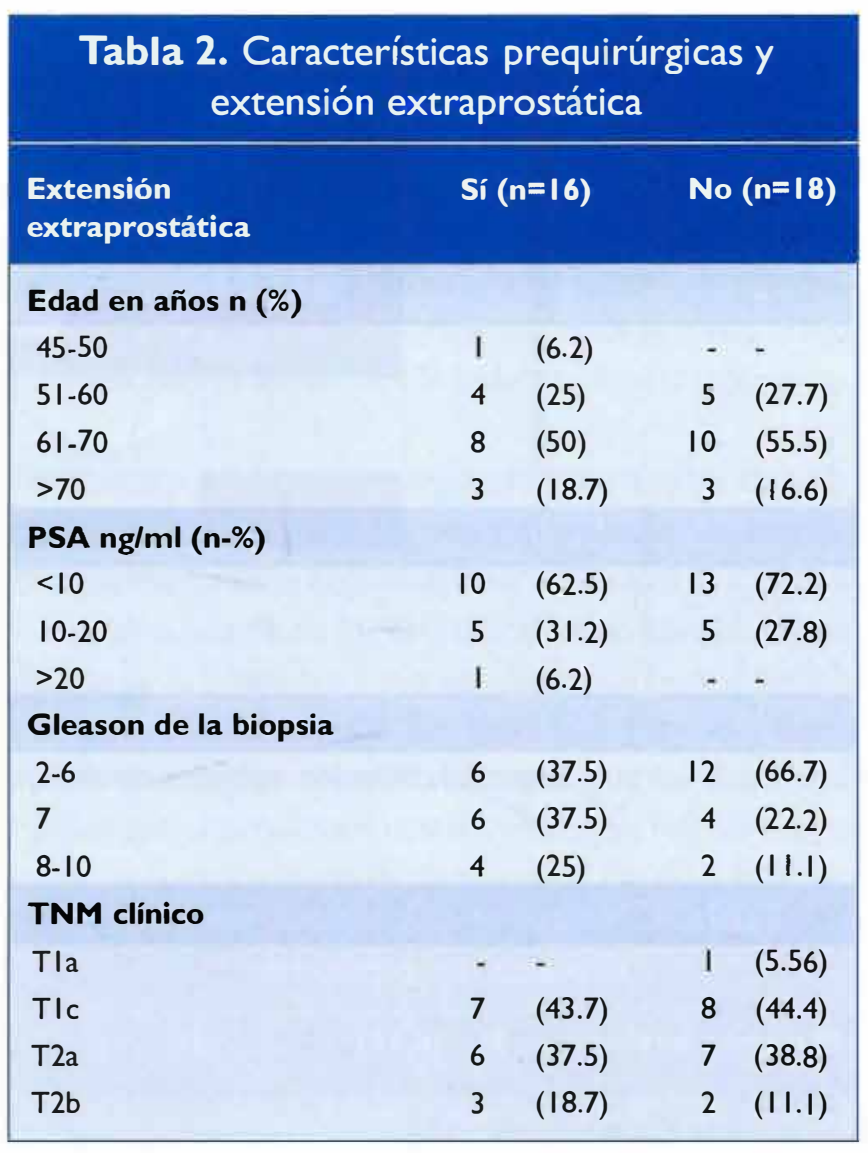

\begin{tabular}{|c|c|c|c|c|}
\hline Márgenes positivos & & $(n=15)$ & & Jo $(n=19)$ \\
\hline \multicolumn{5}{|l|}{ Edad en años (n-\%) } \\
\hline $45-50$ & - & - & 1 & (5.3) \\
\hline $51-60$ & 3 & 20 & 6 & $(31.5)$ \\
\hline $61-70$ & 9 & 60 & 9 & $(47.3)$ \\
\hline$>70$ & 3 & 20 & 3 & $(15.7)$ \\
\hline \multicolumn{5}{|l|}{ PSA ng/ml (n-\%) } \\
\hline$<10$ & 10 & $(66.7)$ & 13 & $(68.4)$ \\
\hline $10-20$ & 4 & (26.7) & 6 & $(31.6)$ \\
\hline$>20$ & I & $(6.6)$ & - & - \\
\hline \multicolumn{5}{|c|}{ Gleason de la biopsia } \\
\hline $2-6$ & 5 & (33.3) & 13 & $(68.4)$ \\
\hline 7 & 5 & 33.3 & 5 & $(26.3)$ \\
\hline $8-10$ & 5 & 33.3 & 1 & $(5.2)$ \\
\hline \multicolumn{5}{|l|}{ TNM clínico } \\
\hline $\mathrm{Tla}$ & - & - & 1 & $(5.3)$ \\
\hline TIC & 4 & $(26.7)$ & 11 & (58.9) \\
\hline $\mathrm{T} 2 \mathrm{a}$ & 8 & $(53.3)$ & 5 & $(26.3)$ \\
\hline$T 2 b$ & 3 & (20) & 2 & $(10.5)$ \\
\hline
\end{tabular}




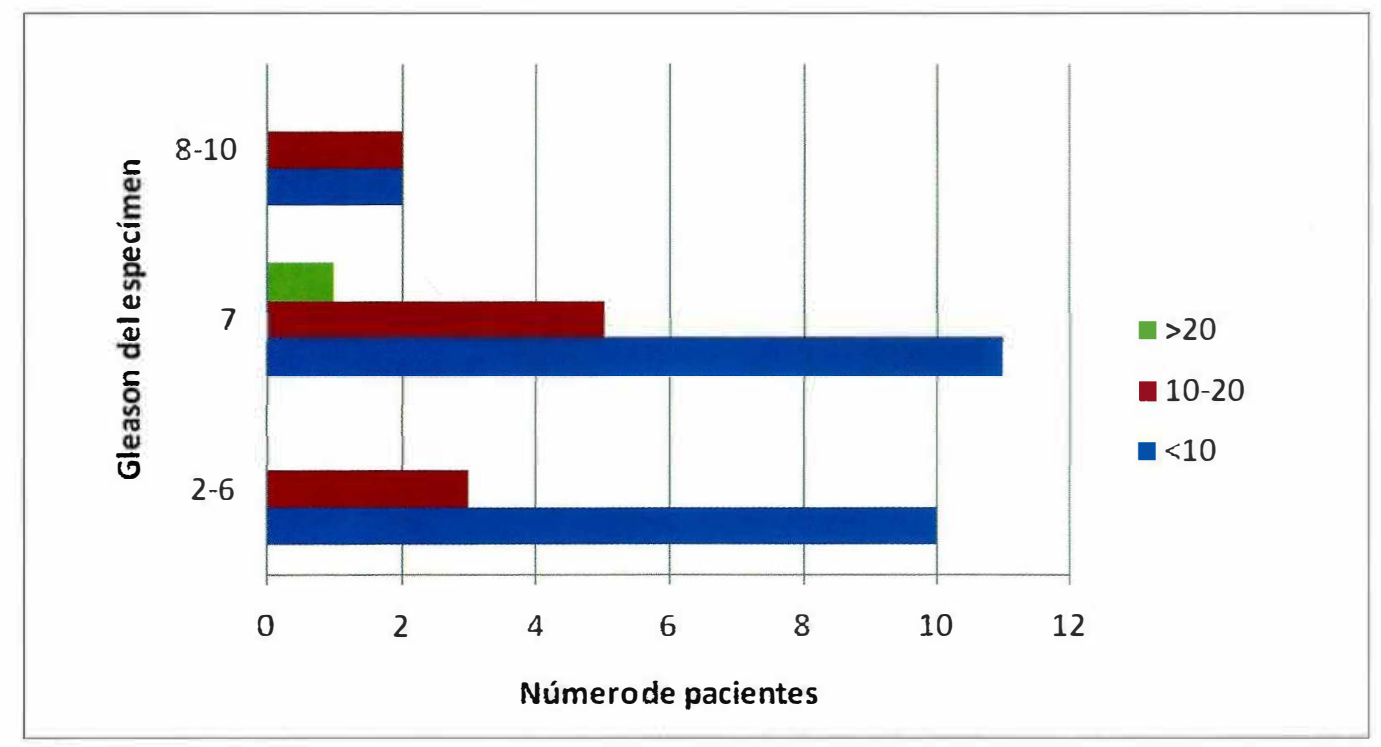

Figura I. Relación entre los niveles séricos de PSA ( $\mathrm{ng} / \mathrm{ml}$ ) y Gleason del espécimen.

\begin{tabular}{|c|c|c|c|c|}
\hline \multicolumn{5}{|c|}{$\begin{array}{l}\text { Tabla 4. Márgenes quirúrgicos p } \\
\text { Gleason del espécimen }\end{array}$} \\
\hline Márgenes positivos & Si & $(n: 15)$ & & (n:19) \\
\hline \multicolumn{5}{|c|}{ Gleason del espécimen, $n$ (\%) } \\
\hline $2-6$ & 4 & (26.7) & 9 & $(47.3)$ \\
\hline 7 & 7 & $(46.6)$ & 10 & (52.6) \\
\hline $8-10$ & 4 & (26.7) & - & \\
\hline
\end{tabular}

Tabla 5. Características prequirúrgicas y extensión extraprostática con márgenes positivos

\begin{tabular}{|c|c|c|c|c|}
\hline $\begin{array}{l}\text { Extensión extraprostática } \\
\text { y márgenes positivos }\end{array}$ & SI & $(n=8)$ & No & $(n=26)$ \\
\hline \multicolumn{5}{|l|}{ Edad en años (n-\%) } \\
\hline $45-50$ & - & - & 1 & (3.8) \\
\hline $51-60$ & 1 & $(12.5)$ & 8 & (30.7) \\
\hline $61-70$ & 6 & (75) & 12 & $(46.1)$ \\
\hline$>70$ & 1 & $(12.5)$ & 5 & $(19.2)$ \\
\hline \multicolumn{5}{|l|}{ PSA ng/ml (n-\%) } \\
\hline$<10$ & 6 & (75) & 17 & $(65.3)$ \\
\hline $10-20$ & 2 & (25) & 8 & (30.7) \\
\hline$>20$ & - & - & I & (3.8) \\
\hline \multicolumn{5}{|l|}{ Gleason de la biopsia } \\
\hline $2-6$ & 2 & (25) & 11 & $(42.3)$ \\
\hline 7 & 4 & (50) & 13 & (50) \\
\hline $8-10$ & 2 & (25) & 2 & (7.7) \\
\hline \multicolumn{5}{|l|}{ TNM clínico } \\
\hline $\mathrm{Tla}$ & - & - & I & (3.8) \\
\hline TIc & 4 & $(50)$ & 11 & $(42.3)$ \\
\hline $\mathrm{T} 2 \mathrm{a}$ & 3 & $(37.5)$ & 10 & $(38.4)$ \\
\hline $\mathrm{T} 2 \mathrm{~b}$ & 1 & $(12.5)$ & 4 & $(15.3)$ \\
\hline
\end{tabular}

\section{Discusión}

El rol del estudio patológico de la biopsia y del espécimen quirúrgico es fundamental para la estadificación y tratamiento de los pacientes con cáncer de próstata. Se definen como factores pronósticos o de riesgo de progresión a ciertas características de las enfermedades que se relacionan con una mayor probabilidad de que a lo largo del tiempo, se produzca un avance en su línea evolutiva. ${ }^{5}$ Las características prequirúrgicas más estudiadas y con mayor relevancia clínica para extensión extraprostática y márgenes quirúrgicos positivos en el espécimen definitivo siguen siendo la edad, los niveles séricos de PSA, el puntaje de Gleason de la biopsia y el TNM clínico.

Más del 70\% de los pacientes con cáncer de próstata son diagnosticados después de 65 años y la mayoría (90\%) de las muertes por esta entidad se presentan en esta población. ${ }^{2,8}$ En nuestro estudio el promedio de edad de los pacientes se encontraba en 64.2 años, sin observar mayor frecuencia de extensión extraprostática y márgenes positivos en los mayores de 70, como la literatura sugiere. Se ha documentado que el tacto rectal es un método de estadificación clínico simple con tendencia a subgraduar la extensión local del cáncer de próstata, lo cual se demostró en nuestro estudio, en donde se observó que aún siendo todos los pacientes clasificados con TNM clínico menor o igual a T2c, 
el 47,1\% de los mismos mostró un TNM patológico mayor o igual a T3 al estudiarse el espécimen de prostatectomía. ${ }^{4,5}$

El PSA es el marcador tumoral más útil del que se dispone en la práctica clínica para el diagnóstico, estadificación, pronóstico y monitorización tras el tratamiento. ${ }^{5}$ Los niveles séricos de PSA en nuestro estudio tuvieron una relación inversa con el puntaje de Gleason del espécimen definitivo, resultados similares a los reportados en estudios anteriores, ${ }^{9}$ debido probablemente a la era de la utilización del PSA como prueba de tamizaje en hombres mayores de 40 años, con el consecuente diagnóstico precoz de la entidad. Los niveles de PSA sérico se correlacionan con cáncer de próstata no localizado y estadios avanzados de la enfermedad.

En las Tablas 2 y 3 se observa que $62,5 \%$ de los pacientes con extensión extraprostática y $66 \%$ con márgenes positivos tienen niveles de PSA menores a $10 \mathrm{ng} / \mathrm{ml}$, en contravía con lo reportado en estudios anteriores. ${ }^{4,5}{ }^{5} \mathrm{La}$ frecuencia de márgenes positivos y extensión extraprostática fue de 44 y $47 \%$. Se observó mayor compromiso de márgenes quirúrgicos y extensión extraprostática en casos de Gleason superior a 8.

El sistema de graduación histológica del cáncer de próstata más utilizado es el puntaje de Gleason. Valores altos se correlacionan con estadios avanzados de la enfermedad. En nuestra serie no hubo mayor frecuencia de Gleason mayor o igual a 8 con extensión extracapsular y márgenes positivos y la mayoría de los estudiados presentaban cifras menores de 7. Estos datos pueden ser el resultado de la actualización de los criterios para clasificación histológica en $2005^{7}$ utilizados en nuestro estudio. Cabe resaltar que los pacientes que presentaron puntaje mayor o igual a 8 mostraron una mayor frecuencia de cáncer de próstata no localizado.

Los factores de riesgo utilizados demuestran ser más útiles si se combinan formando grupos, teniendo en cuenta el peso de cada variable, comparados con su estimación pronóstica de modo individual, lo cual se demuestra en la utilidad de los nomogramas y tablas de predicción. ${ }^{10}$
La limitación de nuestro estudio es la pequeña población, debido a datos incompletos en la historia clínica y en particular a la pérdida de centralización del material para estudio histopatológico, lo cual afecta el análisis y la interpretación de los datos. Sin embargo, el uso de nomogramas para la predicción de cáncer de próstata no localizado es relevante y sigue siendo recomendado, permitiendo el ajuste de las indicaciones terapéuticas.

En nuestro medio no se encontraron estudios de validación de dichos nomogramas y el realizado en nuestra institución en años anteriores solo encontró el T3 como factor predictor con significancia estadística, ${ }^{6}$ lo cual difiere de la literatura y con esta serie, en la cual ningún paciente con T3 clínico fue llevado a prostatectomía radical. Se sugiere la realización de nuevas investigaciones con una población suficiente que brinde mayor peso en el análisis estadístico, con el fin de establecer una relación más fuerte entre las variables.

\section{Referencias}

1. Jemal A, Siegel R, Ward E, Murray T, Xu J, Smigal C, et al. Cancer statistics, 2006. CA Cancer Journal for Clinicians. 2006 Mar;56(2):106-30.

2. Eble JN, Sauter G, Epstein JE. Prostate carcinome. World Health Organization Classification of Tumours. Pathology and Genetics of Tumours of the Urinary System and Male Genital Organs. Lyon, France: World Health Organization. $2004 ; 159$.

3. Buhmeida A, Pyrhonen S, Laato M, Collan Y. Prognostic factors in prostate cancer. Diagnostic Pathology. 2006 Apr 3;1(4):4.

4. Partin A, Yoo J, Carter H, Pearson J, Chan D, Epstein J, et al. The use of prostate specific antigen, clinical stage and Gleason puntaje to predict pathological stage in men with localized prostate cancer. The Journal of Urology. 1993;150(1):110-4.

5. Segarra TJ, Millan RF, Palou RJ, Villavicencio MH. [Prognostic factor and prediction tables for clinically localized prostate cancer]. Actas Urológicas Españolas. 2006 Jun;30(6):567-73

6. Aponte H, Hernández F, Yaspe E, Méndez L. Factores prequirúrgicos predictores de extensión extracapsular y márgenes positivas, en pacientes con adenocarcinoma de próstata, tratadas mediante prostatectomía radical en el Hospital Universitario de San José, Bogotá, Colombia. Revista Colombiana de Urología. 2001;10(3):102-5.

7. Epstein JI, Allsbrook WC, Jr., Amin MB, Egevad LL. The 2005 International Society of Urological Pathology (ISUP) Consensus Conference on Gleason Grading of Prostatic Carcinoma. American Journal of Surgical Pathology. 2005 Sep;29(9):1228-42.

8. Routh JC, Leibovich BC. Adenocarcinoma of the prostate: epidemiological trends, screening, diagnosis, and surgical management of localized disease. Mayo Clinic Proceedings. 2005 Jul;80(7):899-907.

9. Aihara M, Levobitz R, Wheeler T, Kinner B, Ohori M, Scardino P. Prostate specific antigen and Gleason grade: an immunohistochemical study of prostate cancer. The Journal of Urology. 1994;151:1558-64

10. Makarov D, Trock B, Humphreys E, Mangold L, Walsh P, Epstein JI, et al. Update normogram to predict pathology stage of prostate cancer given prostatespecific antigen level, clinical stage and biopsy Gleason puntaje (Partin tables) based on cases from 2000 to 2005. Urology. 2007;69(6):1095-101 